\title{
Fluorescence single particle tracking for sizing of nanoparticles in undiluted biological fluids
}

\author{
Kevin Braeckmans ${ }^{1}$, Kevin Buyens ${ }^{1}$, Wim Bouquet-Geerardyn ${ }^{+}$, Chris Vervaet $^{+}$, Philippe Joye ${ }^{0}$, Filip De \\ Vos $^{0}$, Laurent Plawinski ${ }^{\S}$, Loïc Doeuvre ${ }^{\S}$, Eduardo Angles-Cano ${ }^{\S}$, Niek N. Sanders ${ }^{\S}$, Jo Demeester*, Stefaan \\ C. De Smedt* \\ * Laboratory of General Biochemistry and Physical Pharmacy, Ghent University, Harelbekestraat 72 , \\ 9000 Gent, Belgium \\ ${ }^{+}$Laboratory of Pharmaceutical Technology, Ghent University, Harelbekestraat 72, 9000 Gent, \\ Belgium \\ ${ }^{\circ}$ Laboratory of Radiopharmacy, Ghent University, Harelbekestraat 72, 9000 Gent, Belgium \\ § Inserm U919, UMR CNRS 6232, GIP Cyceron, Bd Henri Becquerel, 14074-cedex, Caen, France \\ ${ }^{\$}$ Laboratory of Gene Therapy, Ghent University, Heidestraat 19, 9820 Merelbeke, Belgium
}

\begin{abstract}
While extremely relevant to many life science fields, such as biomedical diagnostics and drug delivery, studies on the size of nanoparticulate matter dispersed in biofluids are missing due to a lack of suitable methods. Here we report that fluorescence single particle tracking (fSPT) with maximum entropy analysis is the first technique suited for accurate sizing of nanoparticles dispersed in biofluids, such as whole blood. After a thorough validation, the ISPT sizing method was applied to liposomes that have been under investigation for decades as nanocarriers for drugs. The tendency of these liposomes to form aggregates in whole blood was tested in vitro and in vivo. In addition, we have demonstrated that the fSPT sizing technique can be used for identifying and sizing natural cell-derived microparticles directly in plasma. fSPT sizing opens up the possibility to systematically study the size and aggregation of endogenous or exogenous nanoparticles in biofluids.
\end{abstract}

Keywords: single particle tracking, particle size, nanoparticles, nanomedicines, fluorescence microscopy, nanobiophotonics

\section{INTRODUCTION}

Detecting submicrometer particles and aggregates in biological fluids is an area of great interest in a broad range of disciplines. One example in the context of biomedical diagnostics is the detection of blood-borne cell derived microparticles which could serve as biomarkers for metabolic and systemic diseases, as well as for thrombosis (myocardial infarction, stroke) and cancer ${ }^{1,2}$. In pharmacy, nanomedicines are being developed to deliver therapeutic agents, such as anti-cancer drugs and nucleic acids, to specific target tissues. The therapeutic compounds are combined with (non-viral) carrier materials to form particles typically in the $50-500 \mathrm{~nm}$ range. Often these nanomedicines should be administered by intravenous (IV) injection, such as liposomes containing doxorubicin, which was one of the first regulatory approved types of nanomedicines. Also in the context of biomedical imaging, nanoparticles in the 1-100 nm range are typically IV injected, such as gold nanoparticles for X-ray computer tomography, iron oxide particles for NMR imaging, quantum dots for fluorescence imaging, etc. ${ }^{3}$ In all those applications, the effective size of the nanoparticles in the blood circulation is extremely important as it is directly related to their clearance and bio-distribution ${ }^{4-6}$. In addition it is known that size directly influences uptake and processing of nanoparticles by target cells ${ }^{7,8}$. 
While extremely relevant for many life science applications, no studies have been reported so far where the size and aggregation of nanoparticles has been studied directly in induluted biological fluids. The main reason why such studies are lacking is that no methods are currently available that allow to do this accurately and reliably. To address this need, we have developed a novel method, based on fluorescence single particle tracking (fSPT) combined with maximum entropy analysis ${ }^{9}$. fSPT is a microscopy technique that allows to image the movement of individual fluorescently labeled molecules or particles with very high precision (typically in the order of $10 \mathrm{~nm}$ ). Here we report that fSPT, when combined with maximum entropy analysis, is the first technique that can accurately measure the size distribution of fluorescently labelled particles directly in complex biological fluids, such as plasma and even whole blood.

\section{MATERIALS AND METHODS}

\subsection{Fluorescent polystyrene nanospheres}

Yellow-green fluorescent carboxylated polystyrene nanospheres of nominal size $0.1 \mu \mathrm{m}$ and $0.2 \mu \mathrm{m}$ were purchased from Invitrogen (Merelbeke, Belgium). The nanospheres were coated with Pluronic F-127, a block-copolymer of polyethyleneglycol (PEG) and polypropylene oxide, which was purchased from Sigma Aldrich (Bornem, Belgium). The coating procedure was carried out as previously described ${ }^{10}$.

\subsection{Liposomes}

Cationic liposomes were prepared from equimolar amounts of the cationic lipid 1,2-dioleyl-3trimethylammoniumpropane (DOTAP) and the neutral fusogenic lipid 1,2-dioleoyl-sn-glycero-3-phosphoethanolamine (DOPE). $0.1 \mathrm{~mol} \%$ DOPE-LissamineRhodamineB $\left(\lambda_{\mathrm{ex}}=557 \mathrm{~nm}, \lambda_{\mathrm{em}}=571 \mathrm{~nm}\right)$ was incorporated into the liposomes for fluorescent labeling. All lipids were purchased from Avanti Polar Lipids (Alabaster, AL). Functionalisation of the DOTAP-DOPE liposomes with polyethylene-glycol (PEG) chains was done through the addition (3,5 and 10 mol\%) of 1,2-distearoyl-sn-glycero-3-phosphoethanolamine- $\mathrm{N}$-[methoxy(polyethylene glycol)-2000] (DSPE-PEG) or $\mathrm{N}$ palmitoyl-sphingosine-1-\{succinyl[methoxy(polyethylene glycol)2000]\} (C16 PEG-Ceramide). To prepare the liposomes, the lipids were dissolved in chloroform and mixed in a round bottomed flask. A lipid film was formed by rotary evaporation of the chloroform at $40{ }^{\circ} \mathrm{C}$. The dried lipid film was rehydrated with $20 \mathrm{mM}$ Hepes buffer $\mathrm{pH} 7.4$, resulting in a final concentration of $5 \mathrm{mM}$ DOTAP, $5 \mathrm{mM}$ DOPE and a suitable concentration of DSPE-PEG or C16 PEG-Ceramide. The lipid film was allowed to hydrate resulting in liposome formation. Finally, the liposomes were extruded 11 times (at room temperature) through a polycarbonate membrane filter with $100 \mathrm{~nm}$ pores (Whatman, Brentfort, UK).

\subsection{Human blood samples}

Li-heparine tubes (Terumo Europe, Leuven, Belgium) were used for collection of blood from healthy volunteers. For plasma preparation, the blood tubes were centrifuged for 30 minutes at $700 \mathrm{~g}$ in a swinging bucket centrifuge (Heraeus Multifuge 1 S-R, DJB Labcare, Newport Pagnell, UK). Human serum was obtained from blood from healthy volunteers freshly collected in Venoject tubes containing a clotting activator and a separation gel (Terumo Europe, Leuven, Belgium). After 30 minutes of clotting, the blood was centrifuged for 30 minutes at $700 \mathrm{~g}$. All sizing experiments were carried out on freshly collected blood samples.

Using a capillary viscosimeter (model PVS1; Lauda, Lauda-Königshofen, Germany), the dynamic viscosity of both human plasma and human serum was found to be constant at approximately $1.35 \mathrm{cP}$ over $24 \mathrm{~h}$ (measured at $37^{\circ} \mathrm{C}$ ) and varied less than $2 \mathrm{cP}$ between different donors. For all SPT measurements a value of $1.35 \mathrm{cP}$ was used. 


\subsection{Fluorescence SPT sizing in whole human blood}

To mimick the blood circulation in rats, an artificial circulation system was set up consisting of tubing with $1.6 \mathrm{~mm}$ inner diameter (Helix medical, Carpinteria, CA, USA) and a peristaltic pump (BL 760 FB, Bellco, Mirandola, Italy). The entire system was put in an incubator at $37^{\circ} \mathrm{C}$. To study liposome aggregation in blood, $200 \mu 1$ of a 1/50 dilution of the liposome stock dispersion was injected into $5 \mathrm{ml}$ of blood in the circulation system. $5 \mu \mathrm{l}$ samples were taken from the blood circulation at regular time intervals and transferred to a microscope slide. The sample thickness was always 120 $\mu \mathrm{m}$ by using using double adhesive spacers (Secure-Seal Spacer, Molecular Probes, Leiden, The Netherlands) in between the microscope slide and cover slip. Fluorescence SPT measurements were subsequently performed on the microscope stage at $37^{\circ} \mathrm{C}$, using an objective heater (Bioptechs, Butler, PA) in combination with a warm stage (Linkam, Waterfield, United Kingdom).

\subsection{DLS measurements}

A Nano-ZS (Malvern; Worcestershire, UK) was used for dynamic light scattering (DLS) size measurements. Zeta potential measurements were performed on the same instrument (in $20 \mathrm{mM}$ Hepes buffer $\mathrm{pH} \mathrm{7.4)} \mathrm{resulting} \mathrm{in} \mathrm{a} \mathrm{zeta}$ potential of around $+50 \mathrm{mV}$ for the non-pegylated liposomes, and around $+15 \mathrm{mV}$ for the pegylated liposomes.

\subsection{Fluorescence SPT size measurements}

A custom-built laser widefield epi-fluorescence microscope set-up was used for the fluorescence SPT measurements. A $100 \mathrm{~mW}$ Calypso $491 \mathrm{~nm}$ laser (Cobolt, Solna, Sweden) was used as the illumination source. The microscope was a Nikon TE2000E (NIKON BELUX, Brussels, Belgium) with a Nikon Plan Apochromat 100× NA1.4 oil immersion objective lens. Digital time-lapse recordings were made with an electron multiplying CCD camera (Cascade II:512; Roper Scientific, Tucson, AZ).

To image the diffusional movement of the nanoparticles, the microscope was always focused between 5 and $10 \mu \mathrm{m}$ from the cover glass to avoid deviations from free diffusion due to the presence of the cover slip. Using custom developed software the SPT movies were analyzed to obtain the motion trajectories of all individual particles. By calculating the diffusion coefficient for each trajectory, a distribution of diffusion coefficients can be obtained which can be transformed to a size distribution with the well-known Stokes-Einstein equation. The raw size measurement is refined by a Maximum Entropy deconvolution process which eliminates statistical broadening of the distribution and sampling noise, as we have recently reported in more detail ${ }^{9}$. In short, the empirical diffusion coefficient $D_{e}$ as determined from the mean square displacement of a single trajectory with $K$ steps, follows a gamma density function according to:

$$
f\left(D_{e}\right) \mathrm{d} D_{e}=\frac{K^{K} D_{e}^{K-1}}{(K-1) ! D^{K}} e^{-K D_{e} / D} \mathrm{~d} D_{e}
$$

where $D$ is the true diffusion coefficient. If $\alpha(D)$ represents the distribution of diffusion coefficients of particles in the dispersion, then the distribution of empirical diffusion coefficients becomes:

$$
f\left(D_{e}\right) \mathrm{d} D_{e}=\int \alpha(D) f_{\gamma}\left(D_{e} ; K, \frac{D}{K}\right) \mathrm{d} D \mathrm{~d} D_{e}
$$

Making the transition to a discrete space (for computational reasons) of diffusion coefficients $\left[D_{1}, \ldots, D_{N}\right]$ with fractions $\left[\alpha_{1}, \ldots, \alpha_{N}\right]$, the corresponding cumulative distribution becomes: 


$$
F\left(D_{e}\right)=\sum_{i=1}^{N} \alpha_{i} \frac{\gamma\left(K, D_{e} K / D_{i}\right)}{(K-1) !}
$$

where $\gamma(a, x)=\int_{0}^{x} t^{a-1} e^{-t} d t$. By measuring the distribution of empirical diffusion coefficients from trajectories of length $K$, Eq. (3) can be used to determine the fractions $\left[\alpha_{1}, \ldots, \alpha_{N}\right]$ of the underlying 'true' distribution of diffusion coefficients by standard least squares fitting. However, to avoid over-interpretation of features in the distribution that are not statistically warranted, an extra criterion of maximum entropy is introduced according to the "maximum entropy method' (MEM) ${ }^{11}$. MEM will basically try to find the best fitting solution with maximum entropy, where entropy $S$ is defined according to Shannon-Jaynes as $S=-\sum_{i=1}^{N} \alpha_{i} \ln \alpha_{i}$.

\section{RESULTS}

\subsection{Validation of fSPT sizing}

From the movies obtained by fSPT, the motion trajectories of fluorescently labeled particles can be obtained, as is illustrated in Figure 1. The diffusion coefficient can be calculated for each trajectory from its mean square displacement, resulting in a distribution of empirical diffusion coefficients, which can then be refined by MEM analysis and resulting in the corresponding size distribution. An example is shown in Figure 1 for fluorescent nanospheres of $100 \mathrm{~nm}$ nominal size dispersed in HEPES buffer. The mean size was $121.1 \mathrm{~nm}$, which compared very well to the value of $121.8 \mathrm{~nm}$ as obtained by dynamic light scattering experiments on the same dispersion. The same nanospheres were dispersed in serum, giving a mean size of $136.5 \mathrm{~nm}$, which is slightly larger than in HEPES buffer, most likely due to serum proteins that adsorb to the nanosphere surface. Note that a reference measurement by DLS in serum is not possible due to the many light scattering components in serum. fSPT is, therefore, the first technique that allows to size (fluorescently labeled) nanoparticles in undiluted biological fluids.
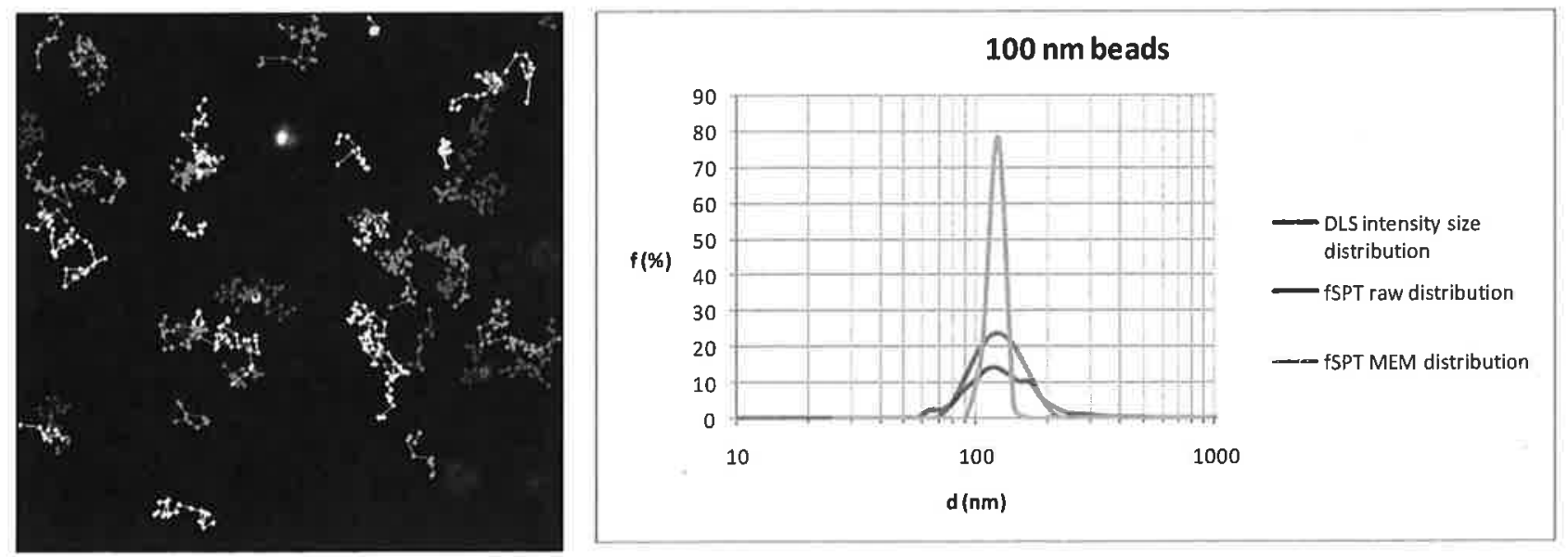

Figure 1. Single particle trajectories are shown as obtained from an ISPT movie of fluorescent nanospheres diffusing in HEPES buffer. The corresponding size distrubutions are shown measured by dynamic light scattering and fSPT. For ISPT the size distribution is shown before and after MEM analysis. 


\section{2 fSPT sizing of liposomes in whole blood}

In a next step we investigated whether we can monitor the size of liposomes, fluorescently labeled by incorporating 0.1 mol\% DOPE-LissamineRhodamineB, in whole blood. Liposomes were prepared with and without polyethylene-glycol (PEG) coating. Covering the surface of nanoparticles with PEG chains is currently the most frequently used approach to increase the blood circulation time. Here we have used ASPT to examine directly for the first time if pegylation suppresses the aggregation of liposomes in whole blood. A first type of pegylated DOTAP-DOPE liposomes was prepared through the addition (10 mol\%) of the PEG containing lipid DSPE-PEG. A second type of pegylated liposomes was obtained using $10 \mathrm{~mol} \%$ ceramide-PEG with a $\mathrm{C} 16$ acyl chain. The liposomes were injected into the artificial blood circulation system and ISPT size measurements were performed on $5 \mu 1$ blood samples at $30 \mathrm{~min}$ time intervals. It is clear from the results in Figure 2 that non-pegylated liposomes strongly aggregate already from the beginning, while the pegylated ones remain stable over a longer time. DSPE-PEG liposomes proved to be more stable then the ceramide-PEG liposomes, which can be explained by the ability of the ceramide-PEG molecules to gradually diffuse out of the liposomes ${ }^{12}$.

\subsection{In vivo experiments}

As ASPT sizing in whole blood proved to be feasible and only requires samples of a few microliter, we proceeded with investigating the size of liposomes by fSPT which were first IV injected in rats. For each of the three types of liposomes, three rats were injected with $200 \mu \mathrm{l}$ of liposome suspension after which small blood samples $(50-100 \mu \mathrm{l})$ were taken in Li-heparin vials at regular time intervals after injection. In accordance with the results from the artificial circulation system, the non-pegylated liposomes showed severe aggregation after 5 minutes (Fig. 3a). At later time points it was found that the larger particles had disappeared from the blood, leaving only the smaller ones. After 60 min only very few liposomes were left and no meaningful size distributions could be obtained anymore. Also for the ceramide-PEG liposomes some aggregation was found at the first time point, which disappeared at later times. These results indicate that larger particles are more rapidly cleared from the blood circulation than smaller ones. The least aggregation was observed for the DSPE-PEG liposomes and the particles remained the longest in the blood circulation. From these in vivo experiments we conclude that the sizing of nanoparticles in blood should at first be performed in a closed artificial blood circulation system since aggregates are quickly removed from the bloodstream in vivo which may lead to an underestimation of the extent of aggregation.

\section{Conclusions}

In this study we have shown that fSPT combined with MEM analysis can accurately measure the size distribution of nanoparticles dispersed in undiluted biological fluids. From experiments with an artificial blood circulation system we found that non-pegylated DOTAP-DOPE liposomes quickly aggregated, while pegylated liposomes were less prone to aggregation. When comparing ceramide-PEG and DSPE-PEG containing liposomes, the latter ones were found to be the most stable due to the ability of the ceramide PEG to gradually diffuse out of the liposomes. From in vivo experiments it became clear that aggregates are more quickly removed from the blood circulation compared to small particles so that the longest circulation time was found for the DSPE-PEG liposomes. Considering these promising first results, we believe that ISPT can become an important tool in pharmacy, biomedical imaging and diagnostics, or in any field where nanomatter has to be characterized in biological fluids. 
a

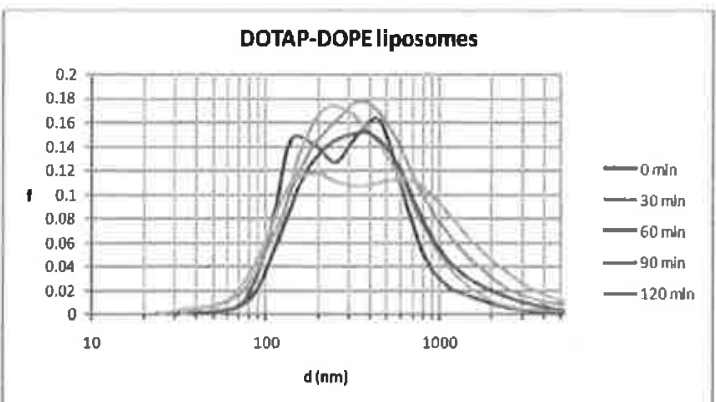

b

DOTAP-DOPEliposomeswith 10 mol\%Ceranide-PEG

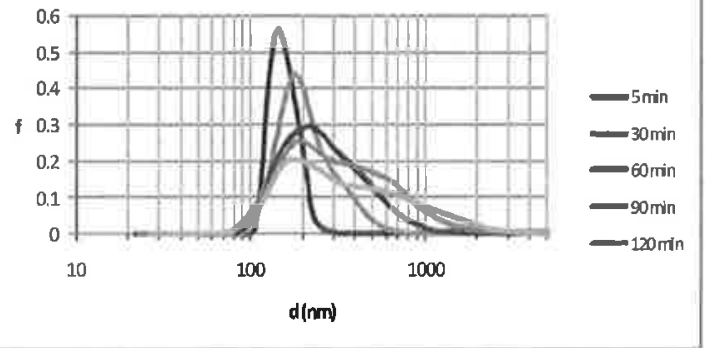

C

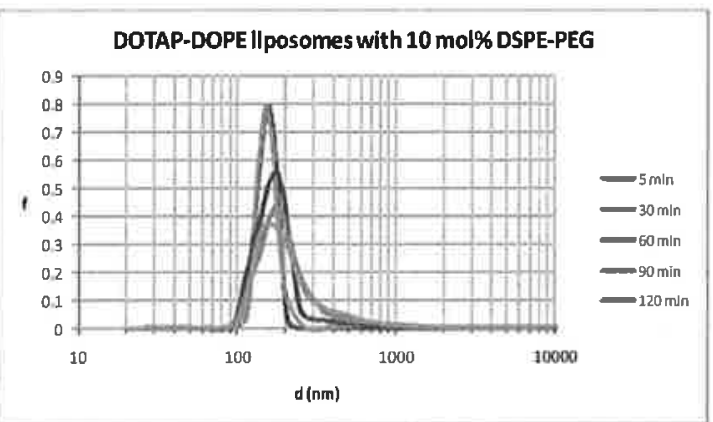

Figure 2. DOTAP-DOPE liposomes with and without PEG coating were sized in whole blood over time. Pegylation of the liposomes was done by incorporating on the one hand 10\% DSPE-PEG into the liposomes, and on the other hand $10 \mathrm{~mol} \%$ of C16 ceramide-PEG. 
a

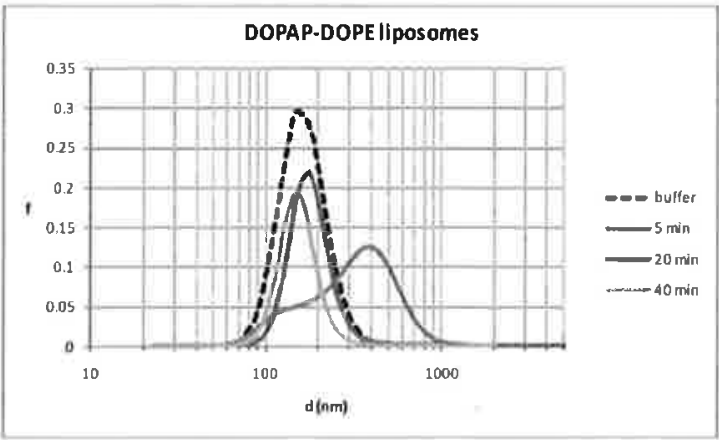

b

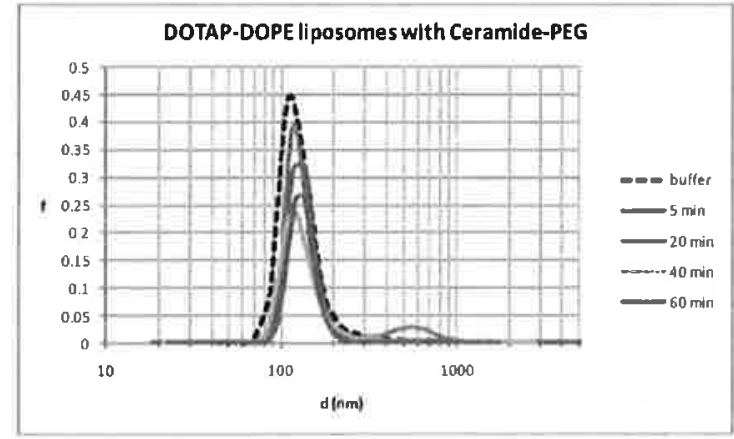

C

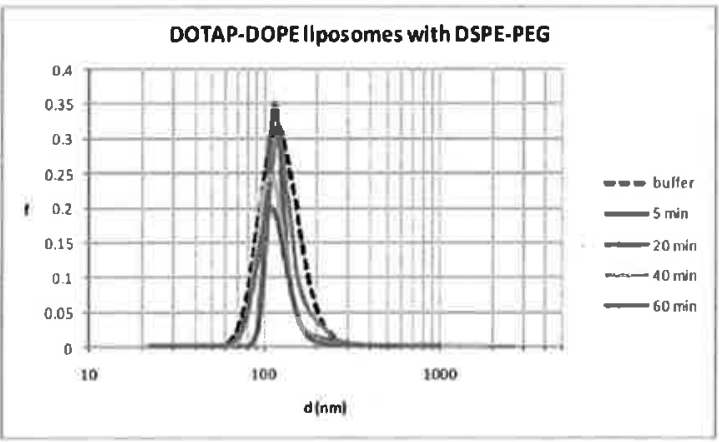

Figure 3. fSPT was used to size DOTAP-DOPE liposomes which were intravenously injected in rats at regular time intervals for ISPT size measurements. (a) Non-pegylated liposomes immediately form large aggregates which are quickly removed from the blood circulation leaving only small non-aggregated liposomes after $20 \mathrm{~min}$. From $60 \mathrm{~min}$ on fSPT sizing was not possible anymore since almost no liposomes were left in the blood circulation. (b) For ceramide-PEG liposomes a limited amount of aggregates was found at early time points which are removed at later times. (c) Liposomes with DSPE-PEG are the most stable over the entire measurement period.

\section{ACKNOWLEDGEMENTS}

Financial support by the Ghent University Special Research Fund and the Fund for Scientific Research Flanders (Belgium) is acknowledged with gratitude. 


\section{References}

[1] Chironi, G.N., Boulanger, C.M., Simon, A., Dignat-George, F., Freyssinet, J.M., Tedgui, A. "Endothelial microparticles in diseases," Cell Tissue Res. 335, 143-151 (2009).

[2] Doeuvre, L., Plawinski, L., Toti, F., Angles-Cano, E. "Cell-derived microparticles: a new challenge in neuroscience," J. Neurochem. 110, 457-468 (2009).

[3] Nune, S.K., Gunda, P., Thallapally, P.K., Lin, Y.Y., Forrest, M.L., Berkland, C.J. "Nanoparticles for biomedical imaging," Expert Opin. Drug Deliv. 6, 1175-1194 (2009).

[4] Decuzzi, P., Godin, B., Tanaka, T., Lee, S.Y., Chiappini, C., Liu, X., Ferrari, M. "Size and shape effects in the biodistribution of intravascularly injected particles," J. Control. Release. 141, 320-327 (2010).

[5] Gaumet, M., Vargas, A., Gurny, R., Delie F. "Nanoparticles for drug delivery: the need for precision in reporting particle size parameters," Eur. J. Pharm. Biopharm. 69, 1-9 (2008).

[6] Nagayasu, A., Uchiyama, K., Kiwada, H. "The size of liposomes: a factor which affects their targeting efficiency to tumors and therapeutic activity of liposomal antitumor drugs," Adv. Drug Deliver. Rev. 40, 75-87 (1999).

[7] Rejman, J., Oberle, V., Zuhorn, I.S., Hoekstra, D. "Size-dependent internalization of particles via the pathways of clathrin-and caveolae-mediated endocytosis," Biochem. J. 377, 159-169 (2004).

[8] van der Aa, M.A.E.M., Huth, U.S., Hafele, S.Y., Schubert, R., Oosting, R.S., Mastrobattista, E., Hennink, W.E., Peschka-Suss, R., Koning, G.A. "Cellular uptake of cationic polymer-DNA complexes via caveolae plays a pivotal role in gene transfection in COS-7 cells," Pharm. Res. 24, 1590-1598 (2007).

[9] Braeckmans, K., Buyens, K., Bouquet, W., Vervaet, C., Joye, P., De Vos, F., Plawinski, L., Doeuvre, L., Angles-Cano, E., Sanders, N.N., Demeester, J., De Smedt S.C. "Sizing nanomatter in biological fluids by fluorescence Single Particle Tracking," Nano Letters 10, 4435-4442 (2010).

[10] Peeters, L., Sanders, N.N., Braeckmans, K., Boussery, K., de Voorde, J.V., De Smedt, S.C., Demeester, J. "Vitreous: a barrier to nonviral ocular gene therapy," Invest. Ophth. Vis. Sci. 46, 3553-3561 (2005).

[11] Livesey, A.K., Brochon, J.C. "Analyzing the Distribution of Decay Constants in Pulse-Fluorometry Using the Maximum-Entropy Method," Biophys. J. 52, 693-706 (1987).

[12] Shi, F.X., Wasungu, L., Nomden, A., Stuart, M.C.A., Polushkin, E., Engberts, J.B.F.N., Hoekstra, D. "Interference of poly(ethylene glycol)-lipid analogues with cationic-lipid-mediated delivery of oligonucleotides; role of lipid exchangeability and non-lamellar transitions," Biochem. J. 366, 333-341 (2002). 